Post-print of: Surface Science volume 606, issues 9-10, May 2012, pages 820-824, doi: 10.1016/j.susc.2012.01.017

\title{
Attenuation lengths of high energy photoelectrons in compact and mesoporous SiO2 films
}

F.J. Ferrer (a),J. Gil-Rostra (b),L. González-García (b), J. Rubio-Zuazo (c),P. Romero-Gómez (d),M.C. López-Santos (e), F. Yubero (b)

a Centro Nacional de Aceleradores (CSIC, U. Sevilla), Av. Thomas A. Edison 7, E-41092 Sevilla, Spain

b Instituto de Ciencia de Materiales de Sevilla (CSIC, U. Sevilla), C/ Américo Vespucio 49, E41092 Sevilla, Spain

cSpLine Spanish CRG Beamline at the ESRF, 6 Rue Jules Horowitz, BP 220, F-38043 Grenoble Cedex 09, France

d ICFO, Av. Carl Friedrich Gauss 3, E-08860 Castelldefels, Barcelona, Spain

e LARN Laboratory, University of Namur (FUNDP), 61 rue de Bruxelles, B-5000 Namur, Belgium

\section{Abstract}

We have experimentally evaluated attenuation lengths (AL) of photoelectrons traveling in compact and micro and mesoporous ( 45\% voids) SiO2 thin films with high (8.2-13.2 keV) kinetic energies. The films were grown on polished Si(100) wafers. ALs were deduced from the intensity ratio of the Si $1 \mathrm{~s}$ signal from the $\mathrm{SiO} 2$ film and Si substrate using the two-peaks overlayer method. We obtain ALs of 15-22 nm and 23-32 nm for the compact and porous $\mathrm{SiO} 2$ films for the range of kinetic energies considered. The observed AL values follow a power law dependence on the kinetic energy of the electrons where the exponent takes the values $0.81 \pm 0.13$ and $0.72 \pm 0.12$ for compact and porous materials, respectively.

\section{Keywords}

Hard x-ray photoemission spectroscopy; Attenuation lengths; Inelastic mean free paths;Mesoporous $\mathrm{SiO} 2$ films

\section{Introduction}

Nanostructured materials are nowadays the basis of the design of new sensor [1] or photonic devices [2] and [3]. They are often formed by microporous and/or mesoporous networks (pores size below 2 and $50 \mathrm{~nm}$, respectively). These materials are used, for example, to fabricate porous structures that will work as host of other molecules [3] and [4] or nanoparticles [5]. The surface chemistry and elemental in-depth composition of these new nanostructured materials is traditionally obtained from standard X-ray photoelectron 
spectroscopy (XPS). However, the probing depth of this technique is very shallow and only the top-most surface region (2-4 $\mathrm{nm}$ depth) is accessed.

Hard X-ray photoemission spectroscopy (HAXPES) is getting an increasing interest because it allows studying thicker and/or buried layers (up to few tens of $\mathrm{nm}$ ) than traditional XPS, keeping the chemical sensitivity. In HAXPES, monochromatic hard X-rays are used as excitation source (photon energies $>3 \mathrm{keV}$ ), so high kinetic energies photoelectrons are excited.

Surface analysis with these high energy photoelectrons has advantages and drawbacks comparing with standard XPS. On the one hand, photoionization cross section is strongly reduced when using hard X-rays. On the other hand, probing depth is enhanced due to the increase of the corresponding electron inelastic mean free paths (IMFP). However, in general, the increase of signal due to the larger probing depths does not compensate the decrease in photoionization cross section in order to keep the same order of sensitivities. This, together with the difficulty to have natural monochromatic hard X-ray sources, is the main reason to use synchrotron beamlines with high photon flux to perform HAXPES experiments. Several reviews on this topic describe in detail the performances and possibilities of this technique [6], [7] and [8].

For elemental quantification purposes, reliable attenuation lengths (AL) or IMFP for high kinetic energy electrons have to be used. Although extrapolation of well-established formulae can be used, there is a strong interest in the experimental evaluation of these quantities to validate (or not) the proposed formulae. This is of special interest when we deal with mesoporous materials, i.e., materials with pores sizes smaller than the expected IMFP for high energy photoelectrons.

It is well recognized that the dependence of IMFP $\lambda$ with the electron kinetic energy $E$ in the 0.2-2.0 keV energy range is well reproduced by the TPP2M predictive formula introduced in Ref. [9]. It is expected that this dependence also holds for electron kinetic energies higher than $2 \mathrm{keV}$, as proposed by recent theoretical work by Tanuma et al. [10], for pure elemental materials. However, only few experimental studies devoted to the determination of IMFP at these high energies have been reported [11], [12] and [13].

The dependence of IMFP with the density of the material has not been explored experimentally. It is worth mentioning that in the late nineties there was a strong debate on the way to introduce the density dependence on predictive IMFP formulae. In fact predictive formulae were proposed [14], in which essentially was stated that the IMFP of an electron traveling in matter is inversely proportional to the atomic density, i.e., a model where a porous medium is considered as voids of vacuum in a continuum of matter. Other authors [9], [15] and [16] proposed models where the IMFP or AL are assumed inversely proportional to the square root of the density. In the latter case, a porous medium is still considered as a continuum, but with lower density. Although the first approximation was considered invalid [16] up to now there is no experimental evidence of it.

In this paper we present the first study on the experimental determination of AL of photoelectrons traveling on compact and micro and mesoporous $\mathrm{SiO} 2$ with kinetic energies 
between 8.2 and $13.2 \mathrm{keV}$ using the two-peak overlayer method described by Seah et al. in a series of papers [17].

\section{Experimental}

Well characterized compact and porous $\mathrm{SiO} 2$ thin films samples were deposited on polished $\mathrm{Si}(100)$ wafers for this study. The compact $\mathrm{SiO} 2$ thin films were produced by reactive magnetron sputtering. The distance between the magnetron target and the sample was $10 \mathrm{~cm}$, with the sample surface facing the magnetron target. These films showed refractive indices at $550 \mathrm{~nm}$ about $1.45 \pm 0.03$, as determined by UV-vis reflectance and spectroscopic ellipsometry, which is consistent with a high compactness of the films. The porous SiO2 films were prepared by electron beam evaporation with glancing angle deposition (GLAD) configuration. The refractive index of these porous films at $550 \mathrm{~nm}$ was $1.25 \pm 0.03$ [18] and [19]. This later value is consistent with a solid comprised by a volume of $55 \%$ of compact $\mathrm{SiO} 2$ and $45 \%$ of voids within their structure (according to the effective medium theory), with significant amount of micro and mesopores, as confirmed by isotherm analysis [3] and [18]. The samples ( $10 \times 5 \mathrm{~mm} 2$ in size) were introduced in the vacuum chamber as deposited. No cleaning procedure was used before their HAXPES characterization.

HAXPES analyses of these samples were performed at the BM25 beamline of the ESRF (Grenoble, France) [20] using a high energy FOCUS electron spectrometer [21]. Photon energies of about 10.0, 12.5 and $15.0 \mathrm{keV}$ were used to excite Si 1s signal of the samples (from the $\mathrm{SiO} 2$ films and the Si substrate). The energy resolution $\triangle \mathrm{E} / \mathrm{E}$ of the beamline was $1.5 \times 10-$ 4. Si 1s photoemitted electrons of about 8.2, 10.7 and $13.2 \mathrm{keV}$ were recorded with spectrometer energy resolution of $1 \mathrm{eV}$. The acceptance angle during HAXPES acquisition was $\pm 5^{\circ}$. The angle of incidence $\alpha$ of the radiation was $85^{\circ}$ with respect to the surface normal and the emission angle $\theta$ was $15^{\circ}$ off normal. The beam size was about $0.5 \times 0.5 \mathrm{~mm} 2$ and the number of photons at the sample was about $2 \times 1011 \mathrm{ph} / \mathrm{s}$.

X-ray reflectometry (XRR) measurements were performed in situ (at the same vacuum chamber that the HAXPES measurements) to characterize the thickness and roughness of the $\mathrm{SiO} 2$ films at exactly the same sample position where the HAXPES characterization was made. XRR is well known technique for determining film thicknesses between 5 and $100 \mathrm{~nm}$ on flat substrates. The thickness $d$ may be deduced via the simple relation $p \lambda r \approx 2 d \sin \alpha$ where $\lambda r$ is the wavelength of the radiation and $p$ an integer. Minima or maxima in the reflected intensity are separated by increases of a unity in p. Fig. 1 shows these measurements for two of the samples. In this case photon energy of $10.0 \mathrm{keV}$ was used. The fringes observed in Fig. 1 are due to interferences produced as the X-rays travel at glancing angles between the substrate and the deposited coatings due to their index contrast. The thickness of the films can be estimated from the position of the minima of these spectra. The insets of the figures show the least squared fits used to determine these thicknesses. They are included in the figure, together with the error bars in their determination. Similar fits were obtained for other samples analyzed in this study. Thus, two porous samples of 27.0 and $35.0 \mathrm{~nm}$ and two compact samples of 27.3 and $55.0 \mathrm{~nm}$ where studied. Note that for the same film thicknesses, there are more visible minima for the compact than for the porous SiO2 samples. This is due to 
their lower roughness. Double check was performed ex-situ with an X'pert PRO diffractometer (PANalytical) equipped with a parabolic mirror and PIXcell detector with similar results.

Evaluation of the ALs was done using the two-peak overlayer method that applies to the determination of $\mathrm{SiO} 2$ thickness on Si wafers as described by Seah et al. in a series of papers [17]. According to these authors, ALs can be obtained from the expression

$$
A L(E)=d /\left(\cos \theta \cdot \ln \left(1+\left(\operatorname{Rexpt}(E) / R_{0}\right)\right)\right) \mathrm{ALE}=\mathrm{d} /(\cos \theta \cdot \ln 1+\operatorname{Rexpt}(\mathrm{E}) / \mathrm{R} 0)
$$

Where $\operatorname{Rexpt}(\mathrm{E})$ is the ISi4 +/ISiO intensity ratio of the Si 1s signals from the SiO2 layer and the $\mathrm{Si}$ substrate in the HAXPES spectra, and RO is the equivalent ratio of intensities, 10, $\mathrm{Si} 4+$ and 10, SiO , from bulk oxide and substrate, respectively. RO can be evaluatedtheoretically [22] accordingto

$R_{0} \approx 0.20 \cdot \rho \mathrm{SiO} \cdot(\lambda \mathrm{SiO} / \lambda \mathrm{Si})$

It is worth mentioning that elastic scattering effects are included in previous expression, where $\rho \mathrm{SiO} 2$ is the density $(\mathrm{g} / \mathrm{cm} 3)$ of the $\mathrm{SiO} 2$ film (evaluated from optical measurements in this work as mentioned before). $\lambda \mathrm{SiO} 2 / \lambda \mathrm{Si}$ corresponds to the ratio of the inelastic mean free path for electrons traveling in the $\mathrm{SiO} 2$ overlayer and $\mathrm{Si}$ substrate. To a good approximation this ratio is a constant, that according to TPP2M gets the values 1.26 and 1.50 for the compact and porous SiO2, respectively. Thus, the theoretical values of RO in Eq. (2) are 0.53 and 0.36 for the compact and porous $\mathrm{SiO} 2$. At this point it is worth mentioning that the use of $\mathrm{RO}=0.93$ has been recommended in the past [17] and [22] for compact SiO2 based on standard XPS measurements. This was mainly justified as a relative lack of intensity at the $\mathrm{Si} 1 \mathrm{~s}$ peak of the metallic state compared to the oxide state due to the corresponding uneven contribution of shake-ups at the first plasmon excitation. Note also that if the IMFP is considered inversely proportional to the density (as it is proposed by other authors [14]), R0 would be independent of the density of the SiO2 layer. We will show bellow the validity of these approximations. Table 1 compiles the values of the parameters used for the quantification.

\section{Results}

Fig. 2 shows HAXPES Si 1s spectra (raw data) corresponding to a porous (left) and compact (right) $\mathrm{SiO} 2$ samples acquired using 10.0, 12.5 and $15.0 \mathrm{keV}$ photon energies. The characteristic $\mathrm{Si} 4+$ and $\mathrm{SiO}$ signal from the SiO2 layer and Si substrate are clearly identified. Note that, as the photon energy increases, the relative intensity of the SiO signal from the substrate increases with respect to the $\mathrm{Si} 4+$ of the $\mathrm{SiO} 2$ overlayer. These two silicon chemical species are separated by $5.5 \pm 0.1 \mathrm{eV}$. Besides, the Drudeplasmon excited at the Si substrate is also clearly visible at $17.2 \pm 0.1 \mathrm{eV}$ lower kinetic energies than the SiO signal. Bulk plasmon in SiO is reported with $16.8 \mathrm{eV}$ energy loss both from reflection electron energy loss [23] and standard photoemission experiments [24]. The difference (about $0.4 \mathrm{eV}$ ) can be ascribed to recoil effect due to the high energy of the emitted electrons, in agreement with theoretical predictions [7]. Note also how the energy resolution degrades as the photon energy increases. This is due to the natural broadening induced by the monochromator of the beamline. The figure also includes the deconvolution of the $\mathrm{Si} 4+$ and SiO components used for the quantification purposes. 
Fig. 3 depicts the calculated experimental AL values for the compact (full symbols; circles, squares) and porous (hollow symbols) $\mathrm{SiO} 2$ films, evaluated from the quantification of the measured Si 1s spectra of the SiO2 thin films according to Eq. (1) and the theoretical RO parameters in Table 1. Fig. 3 also includes the evaluation of AL for the compact (full stars) and porous (hollow stars) $\mathrm{SiO} 2$ samples considering $\mathrm{RO}=0.93$. These $\mathrm{AL}$ are about the largest (between 15 and $35 \mathrm{~nm}$ ) experimental AL values ever reported for photoelectrons with kinetic energies in the 8-13 keV. The error bars included in the figure correspond to deviations of \pm $10 \%$ in the R0 values. This figure also includes theoretical IMFP obtained from extrapolation for high kinetic energies of the TPP2M formula [9] for the compact (full line) and porous (dashed line) SiO2 films considered in this work.

The experimental AL values obtained for the compact SiO2 samples, are $~ 10 \%$ lower $(\mathrm{RO}=$ 0.53 ) or $~ 17 \%$ higher $(R 0=0.93)$ than the prediction of the TPP2M formula. In case of porous $\mathrm{SiO} 2$ samples the experimental $\mathrm{AL}$ values are $\sim 4 \%$ lower $(\mathrm{RO}=0.36)$ or $\sim 58 \%$ higher $(\mathrm{RO}=$ $0.93)$ than prediction. Note that for the kinetic energies considered in this work, IMFP and AL differ less than $2 \%$ from each other [22].

The data in Fig. 3 are presented in log-log scale to stress the power law dependence on kinetic energy of $A L$ (i.e., $A L$, IMFP $\propto E m$, where the exponent $m$ corresponds to the slope of the corresponding least squared fit). We obtain that the $\mathrm{m}$ values for the experimental observations are $0.81 \pm 0.13$ and $0.72 \pm 0.12$ for compact and porous materials, respectively (considering RO values from Table 1). The error bars describe the uncertainties in fitting 6 data points ( 2 samples, 3 energies) for each type of material. These values are slightly lower than $m$ $=0.86$, the value predicted by the TPP2M formula for these films.

\section{Discussion}

Strictly speaking the two-peaksoverlayer method is used to determine the effective attenuation length of electrons traveling in matter. However, due to the weak influence of the elastic scattering on $\mathrm{Si}$ and $\mathrm{SiO} 2$ materials when high kinetic energies of the photoelectrons are considered [22] and [25], the values of the corresponding effective attenuation lengths and inelastic mean free path do not deviate significantly (less than $2 \%$ ) from each other. In fact this deviation is smaller than other sources of uncertainties in the quantification described above. This is the reason why, in this particular case, the AL values included in Fig. 3 can also be considered as IMFP.

We have observed that by incorporating 45\% voids in a mesoporous structure to $\mathrm{SiO} 2$, the IMFP increases by $\sim 24 \%$ with respect to that of the compact SiO2 material if theoretical RO values are considered. This result is in reasonable agreement with the predictions of TPP2M (an 20\% increase). Besides the absolute AL values obtained for the compact SiO2 samples, are close to those predicted by TPP2M formula ( 10\% and 4\% lower than the prediction for compact and porous $\mathrm{SiO} 2$ films, respectively).

If IMFP is considered inversely proportional to the material density (i.e., to a good approximation RO does not depend on density), for a SiO2 film with an $\sim 45 \%$ decrease in density with respect to a compact $\mathrm{SiO} 2$ film, the corresponding IMFP would be $\sim 45 \%$ larger. If we assume R0 $=0.93$ (as in the series of papers of Seah et al. [17] and [22]), an 85\% increase 
of IMFP is experimentally obtained (difference between full and hollow stars in Fig. 3). Besides, the absolute AL values obtained under this assumption are significantly larger than those predicted by TPP2M formula ( 17\% and $\sim 58 \%$ higher than the prediction for compact and porous SiO2 films).

Considering these results and that in previous studies was found reasonable agreement between experimental finding of AL [26] and TPP2M predictions of compact SiO2, the results reported in Fig. 3 indicate that the use of TPP2M formulae can safely be extrapolated not only to high kinetic electron energies (up to $13 \mathrm{keV}$ ) but also to micro-mesoporous materials with same chemistry but different density. This is an important result because it constitutes the first experimental evidence of the validity of TPP2M for mesoporous materials.

Among the possible sources of uncertainties in the performed analysis we can name surface contamination, error bars in the determination of thin film thickness and densities, instrumental error bars linked to the experimental set-up and data analysis, and those related to the value of $\mathrm{RO}$ used in the quantification procedure.

As it is mentioned in the experimental section, no especial cleaning procedure was used to remove spurious contamination present at the surface of the samples. Due to the fact that the samples were prepared ex-situ, some carbonaceous contamination (according to standard XPS analysis, $\sim 1 \mathrm{~nm}$ thick) is present at the sample surface. However, we expect this contamination affect evenly to both the signal from the Si substrate and the SiO2 overlayer, so the $\operatorname{Rexpt}(\mathrm{E})$ ratio used in the evaluation of the AL would not be significantly affected.

We also expect minor effects regarding the error bars linked to the experimental set-up. More critical are the uncertainties related to data analysis (determination of the $\operatorname{Rexpt}(\mathrm{E})$ ratio from the area of the deconvoluted Si 1s peak contribution to the HAXPES spectra). Note for example that these error bars increase as the experimental energy resolution degrades, as it is the case for the highest photon energies considered in this study. Fortunately, Fig. 2 shows that the Si4 + and SiO are clearly separated, so we do not expect that data analysis be major concern. Another source of uncertainty in $\operatorname{Rexpt}(E)$ is the excitation of intrinsic plasmons as shake-ups away of the main Si 1s peak intensity. This contribution to the Si 1s emission might be different in metallic $\mathrm{Si}$ and $\mathrm{Si} 4+$ of $\mathrm{SiO} 2$ due to the different screening mechanisms in both oxidations states. It is worth mentioning that this effect has been claimed as the main responsible to use $\mathrm{RO}=0.93$ to quantify by standard XPS compact $\mathrm{SiO} 2$ films with thicknesses below $8 \mathrm{~nm}$ on Si wafers, a value that is significantly higher than its theoretical estimation (c.f. Table 1).

Precise knowledge of the thickness is another issue in the quantification described previously. Uncertainties in this quantity affect directly to the AL values. They were obtained from XRR analysis, which is a robust technique for this purpose for the range of thicknesses considered here. In fact, double check of this analysis was done ex-situ with a commercial XRR spectrometer with similar results within 3\%. We expect that the error bars affecting the AL quantification are about $3 \%$.

Other critical point is the precise control of the density of the films. In our case, it was obtained from the optical analysis of the films (i.e., from their refractive indices) that, together with the effective medium theories, allow to estimate their density. These optical methods are very 
robust to get the refractive index of very thin films when their thickness is known from another technique. The porous films studied in this work were produced and analyzed in a similar manner as in Ref.[18] where a precise control of the optical response is claimed (error bars of $\pm 5 \%$ of the reported density values). However it is worth mentioning that the density in the initial stages of growth of GLAD films (first $~ 50 \mathrm{~nm}$ ) might be higher than the average density of thicker films [19]. In any case, we can consider the density values use in the previous analysis as a lower limit for the porous $\mathrm{SiO} 2$ films, and therefore, the corresponding $\mathrm{AL}$ as higher limit values.

Uncertainties in the values of RO used in Eq. (2) play also a strong role in the quantification. The value of RO to be used in the analysis might be strongly affected [27] (up to a 30\%) by forward focusing effects that are strongly enhanced when the detection is performed in a low index direction of a crystal and low photoelectron kinetic energies. In our case, the HAXPES data acquisition was made at $15^{\circ}$ off normal emission angle to be away from the low index [100] direction. We included in Fig. 3 error bars of $\pm 10 \%$ to account for this effect.

Another possible source of uncertainty is the excitation of surface plasmons by the high energy photoelectrons. The presence of extra surface losses [28] due to the multiple crossing of the electrons through the mesoporous structure (note that the AL in this case are significantly larger than the pore size) would tend to decrease the observed AL with respect to that predicted by IMFP predictive formulae that in general do not include surface losses. This effect would decrease the difference between AL from compact and porous films. Experimentally we observe the opposite behavior ( $\mathrm{AL}$ of the porous $\mathrm{SiO} 2$ samples increase by 24\% with respect to that of the compact SiO2 material while the TPP2M formula predicts an 20\% increase), so we may conclude that surface effects do not seem to be major contribution to the AL, despite the fact that the photoelectrons travel through a series of vacuum-solid interfaces due to the mesoporous nature of the films.

The TPP2M formula, although it is never mentioned in the series of papers where it is introduced, is intrinsically considered to be applied for continuous solids. The density parameter used in the formula is implicitly assumed as that of the particular compact material. According to the results obtained in this work, it reproduces reasonably well the density dependence for mesoporous $\mathrm{SiO} 2$ materials. We are aware that a more thorough analyses need to be performed on this topic, but these first results can be used as a guide for future works.

\section{Conclusions}

We have evaluated experimentally $A L$ of photoelectrons traveling in compact and micro and mesoporous $\mathrm{SiO} 2$ films. The obtained AL (or IMFP) are 15-22 nm for compact SiO2 and 23-32 $\mathrm{nm}$ for porous (45\% voids) SiO2 films for electron kinetic energies between 8.2 and $13.2 \mathrm{keV}$. They are among the highest reported from experimental HAXPES measurements. We have observed that extrapolation of the predictive TPP2M formula to high kinetic energies and low density materials reproduce rather well (both in energy and density dependence) the experimental observations.

\section{Acknowledgments}


We thank the Spanish MICINN (projects MAT2010-18447 and Consolider CSD2008-00023 and CPAN CSD2007-42) for financial support. We also acknowledge MICINN and CSIC for provision of synchrotron radiation facilities and the BM25-Spline staff for the technical support. 


\section{References}

[1]J.R. Sánchez-Valencia, F.J. Aparicio, J.P. Espinós, A.R. González-Elipe, A. Barranco

Phys. Chem. Chem. Phys., 13 (2011), p. 7071

[2]P. Castillero, J.R. Sánchez-Valencia, M. Cano, J.M. Pedrosa, J. Roales, A. Barranco, A.R. González-Elipe

Appl. Mater. Interfaces, 2 (2010), p. 712

[3]L. González-García, I. González-Valls, M. Lira-Cantu, A. Barranco, A.R. González-Elipe

Energy Environ. Sci., 4 (2011), p. 3426

[4]J.R. Sánchez-Valencia, J. Toudert, A. Borrás, C. López-Santos, A. Barranco, I.O. Feliu, A.R. González-Elipe

Plasmonics, 5 (2010), p. 241

[5]M. Cano, P. Castillero, J. Roalesa, J. M. Pedrosa, S. Brittle, T. Richardson, A. R. GonzálezElipe, and A. Barranco, Sens. Actuators, B.150 (2010) 764.

[6]K. Kobayashi

Nucl.Instrum.Methods Phys. Res. A, 601 (2009), p. 32

[7]L. Kover

J. Electron Spectrosc. Relat.Phenom., 178-179 (2010), p. 241

[8]J. Zegenhagen, B. Detlefs

J. Electron Spectrosc. Relat.Phenom., 178-179 (2010), p. 258

[9]S. Tanuma, C.J. Powell, C.R. Penn

Surf. Interface Anal., 21 (1993), p. 165

[10]S. Tanuma, C.J. Powell, C.R. Penn

Surf. Interface Anal., 43 (2011), p. 689

[11]C. Dallera, L. Duò, L. Braicovich

Appl. Phys. Lett., 85 (2004), p. 4532 
[12]M. Sacchi, F. Offi, P. Torelli, A. Fondacaro, C. Spezzani, M. Cautero, G. Cautero, S. Huotari, M. Grioni, R. Delaunay, M. Fabrizioli, G. Vankó, G. Monaco, G. Paolicelli, G. Stefani, G. Panaccione

Phys. Rev. B, 71 (2005), p. 155117

[13]J. Rubio-Zuazo, G.R. Castro

J. Electron Spectrosc. Relat.Phenom., 184 (2011), p. 384

[14]W.H. Gries

Surf. Interface Anal., 24 (1996), p. 38

[15]P.J. Cumpson, M.P. Seah

Surf. Interface Anal., 25 (1997), p. 430

[16]S. Tanuma, C.J. Powell, D.R. Penn

Surf. Interface Anal., 25 (1997), p. 25

[17]M.P. Seah, W.E.S. Unger, H. Wang, W. Jordaan, Th. Gross, J.A. Dura, D.W. Moon, P. Totarong, M. Krumrey, R. Hauert, M. Zhiqiang

Surf. Interface Anal., 41 (2009), p. 430

[18]L. González-García, G. Lozano, A. Barranco, H. Míguez, A.R. González-Elipe

J. Mater. Chem., 20 (2010), p. 6408

[19]L. González-García, A. Barranco, A. Muñoz-Páez, A.R. González-Elipe

Chemphyschem, 11 (2010), p. 2205

[20]J. Rubio-Zuazo, G.R. Castro

Nucl.Instrum. Methods Phys. Res., Sect. A, 547 (2005), p. 64

[21]J. Rubio-Zuazo, M. Escher, M. Merkel, G.R. Castro

Rev. Sci. Instrum., 81 (2010), p. 043304

[22]M.P. Seah, S.J. Spencer

Surf. Interface Anal., 33 (2002), p. 640

[23]F. Yubero, S. Tougaard, E. Elizalde, J.M. Sanz

Surf. Interface Anal., 20 (1993), p. 719

[24]A. Cohen Simonsen, F. Yubero, S. Tougaard

Surf. Sci., 436 (1999), p. 149 
[25]M.P. Seah, I.S. Gilmore

Surf. Interface Anal., 31 (2001), p. 835

[26]S. Tanuma, C.J. Powell, D.R. Penn

Surf. Interface Anal., 17 (1991), p. 927

[27]K.J. Kim, M.P. Seah

Surf. Interface Anal., 39 (2007), p. 512

[28]R.H. Ritchie

Phys. Rev., 106 (1957), p. 874 
Figure captions

Figure 1.XRR spectra (reflected photon intensity vs incidence angle $\alpha$ ) of a compact (a) and a porous (b) samples. The insets show the least squared fit of the position of the minima against the integer $p$ (see text) used to determine the thicknesses $d$ of the films.

Figure 2.Si 1s spectra acquired with 15.0 (top), 12.5 (middle) and $10.0 \mathrm{keV}$ (bottom) photon energies of a porous (left) and compact (right) SiO2 samples deposited on $\mathrm{Si}(100)$ wafers.

Figure 3.Attenuation lengths (AL) obtained after analysis with the two peaks overlayer method of the HAXPES measurements on compact (full symbols) and porous (hollow symbols) $\mathrm{SiO} 2$ films (see text). The error bars correspond to assume $a \pm 10 \%$ uncertainty in the RO values. Full line (dash line) corresponds to the prediction of the extrapolated IMFP values of TPP2M formula for compact (porous) SiO2. 
Table 1

Table 1. Parameters used in the AL quantification from HAXPES measurements according to Eq. (2)[22].

\begin{tabular}{||l|l|l|l||}
\hline & $\boldsymbol{\rho}_{\mathrm{SiO} 2}\left(\mathrm{gr} / \mathbf{c m}^{\mathbf{3}}\right)$ & $\lambda_{\mathrm{SiO} 2} / \lambda_{\mathrm{Si}}$ & $\boldsymbol{R}_{\boldsymbol{0}}$ \\
\hline Compact $\mathrm{SiO}_{2}$ & 2.19 & 1.26 & $0.53 \pm 0.05$ \\
\hline Porous $\mathrm{SiO}_{2}$ (45\% voids) & 1.21 & 1.50 & $0.36 \pm 0.04$ \\
\hline
\end{tabular}


Figure 1

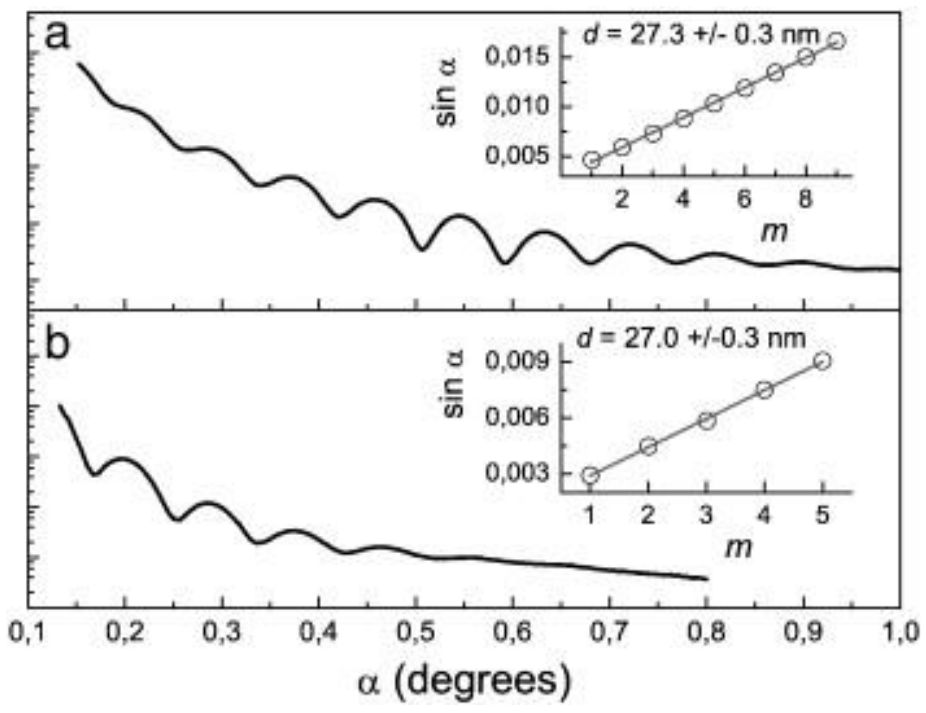


Figure 2

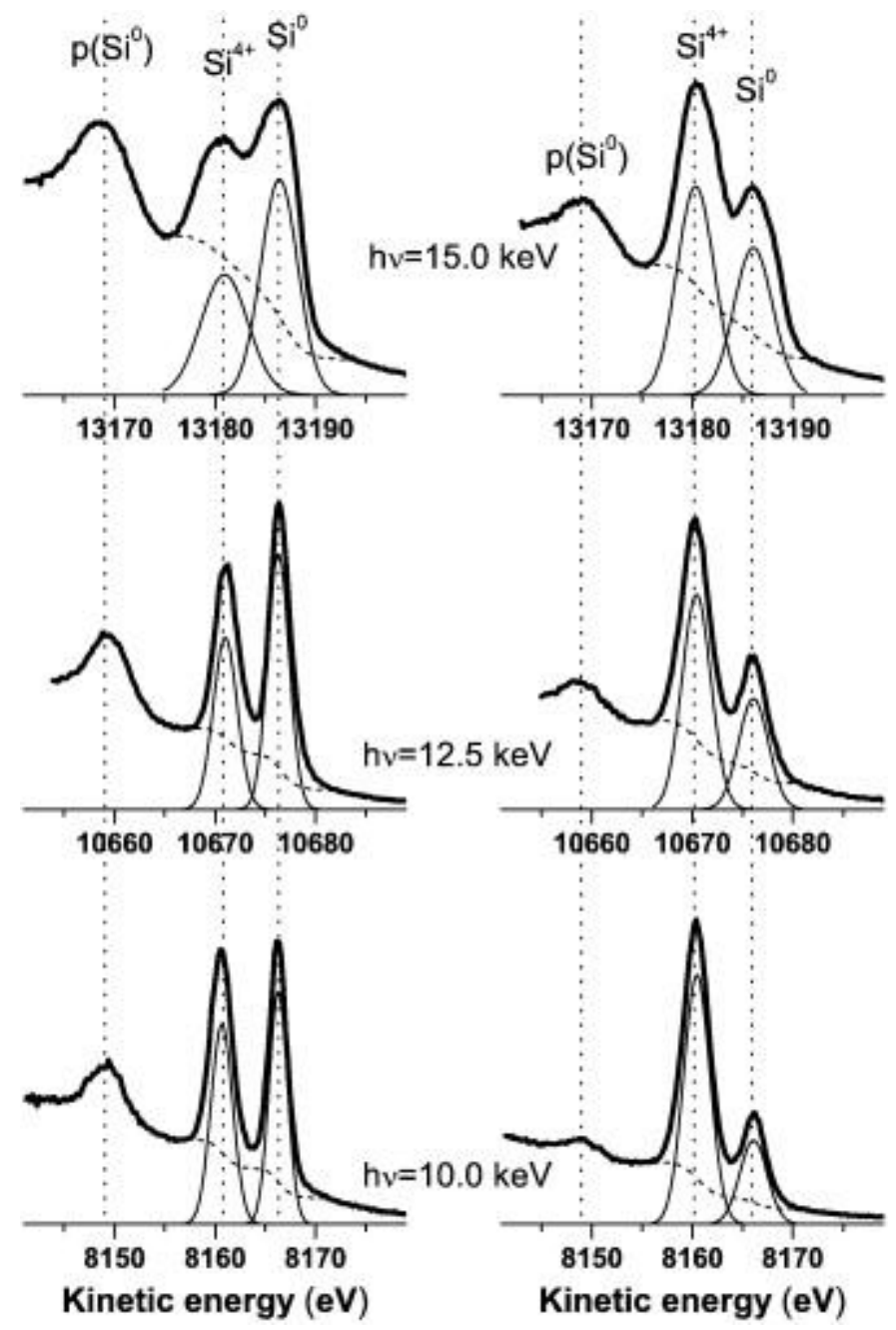


Figure 3

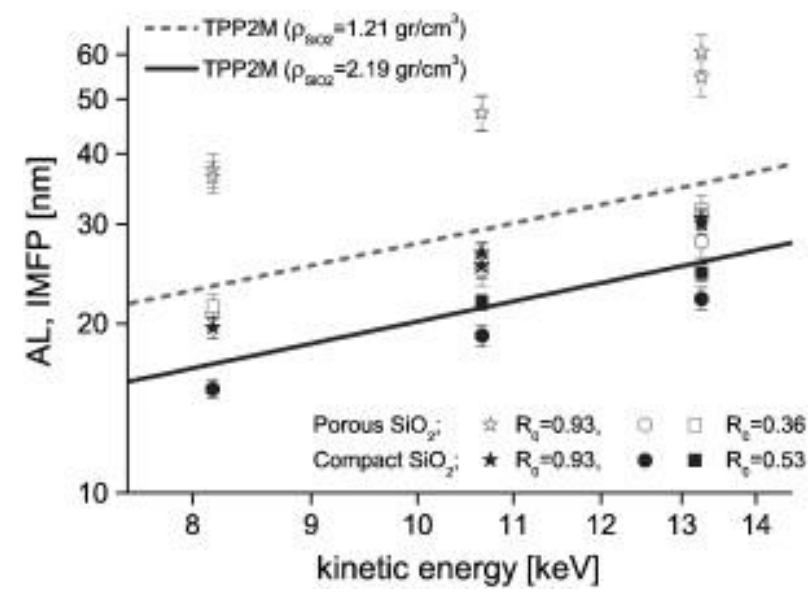

